\title{
Kallikrein 4 (hK4) and prostate-specific antigen (PSA) are associated with the loss of E-cadherin and an epithelial-mesenchymal transition (EMT)-like effect in prostate cancer cells
}

\author{
$T L$ Veveris-Lowe ${ }^{1,2 *}, M$ G Lawrence ${ }^{1 *}, R$ L Collard $^{1 *}$, L Bui $^{1}$, A C Herington ${ }^{1}$, \\ $D L$ Nicol $^{2,3}$ and $J$ A Clements ${ }^{1,2}$
}

\author{
${ }^{1}$ School of Life Sciences and Science Research Centre, Queensland University of Technology, Brisbane, Australia \\ ${ }^{2}$ Wesley Research Institute, Brisbane, Australia \\ ${ }^{3}$ Department of Urology, Princess Alexandra Hospital, Brisbane, Australia \\ (Requests for offprints should be addressed to J Clements, Prostate Cancer Research Program, School of Life Sciences, \\ Queensland University of Technology, GPO Box 2434, Brisbane, 4001, Australia; Email: j.clements@qut.edu.au) \\ * (T L Veveris-Lowe, M G Lawrence and R L Collard contributed equally to this work)
}

\begin{abstract}
Prostate-specific antigen (PSA) and the related kallikrein family of serine proteases are current or emerging biomarkers for prostate cancer detection and progression. Kallikrein $4(K L K 4 / \mathrm{hK} 4)$ is of particular interest, as KLK4 mRNA has been shown to be elevated in prostate cancer. In this study, we now show that the comparative expression of hK4 protein in prostate cancer tissues, compared with benign glands, is greater than that of PSA and kallikrein $2(K L K 2 / h K 2)$, suggesting that $\mathrm{hK} 4$ may play an important functional role in prostate cancer progression in addition to its biomarker potential. To examine the roles that $\mathrm{hK} 4$, as well as PSA and hK2, play in processes associated with progression, these kallikreins were separately transfected into the PC-3 prostate cancer cell line, and the consequence of their stable transfection was investigated. PC-3 cells expressing hK4 had a decreased growth rate, but no changes in cell proliferation were observed in the cells expressing PSA or hK2. hK4 and PSA, but not hK2, induced a 2.4-fold and 1.7-fold respective increase, in cellular migration, but not invasion, through Matrigel, a synthetic extracellular matrix. We hypothesised that this increase in motility displayed by the hK4 and PSA-expressing PC-3 cells may be related to the observed change in structure in these cells from a typical rounded epitheliallike cell to a spindle-shaped, more mesenchymal-like cell, with compromised adhesion to the culture surface. Thus, the expression of E-cadherin and vimentin, both associated with an epithelialmesenchymal transition (EMT), was investigated. E-cadherin protein was lost and mRNA levels were significantly decreased in PC-3 cells expressing hK4 and PSA (10-fold and 7-fold respectively), suggesting transcriptional repression of E-cadherin, while the expression of vimentin was increased in these cells. The loss of E-cadherin and associated increase in vimentin are indicative of EMT and provides compelling evidence that $\mathrm{hK} 4$, in particular, and PSA have a functional role in the progression of prostate cancer through their promotion of tumour cell migration.
\end{abstract}

Endocrine-Related Cancer (2005) 12 631-643

\section{Introduction}

Prostate cancer is now the most frequently diagnosed cancer in men, excluding skin cancers (Jemal et al.
2004). In 2004, it was expected to contribute to $33 \%$ of all newly diagnosed cancers and $10 \%$ of estimated cancer deaths in the USA (American Cancer Society 2004, Jemal et al. 2004). Prostate-specific antigen 
(PSA), also known as kallikrein $3(K L K 3 / \mathrm{hK} 3)$, is a serine protease secreted by normal and malignant prostatic epithelial cells and is the current serum biomarker for prostate cancer (Partin et al. 2002a,b). Kallikrein $2(K L K 2 / \mathrm{hK} 2)$ is also emerging as a useful adjunct marker clinically, particularly in the discrimination of benign and malignant disease in the PSA 4-10 $\mathrm{ng} / \mathrm{ml}$ range, as well as in organ-confined disease (Haese et al. 2001, 2003). Although less widely studied, kallikrein $4(K L K 4 / \mathrm{hK} 4)$ is also highly expressed by the prostate epithelium and is increased in prostate cancer at the mRNA level, suggesting a similar biomarker potential (Obiezu et al. 2002, Xi et al. 2004). Most research has focused principally on the clinical utility of the prostate-specific kallikreins, but much less is known about their biological role in prostate cancer progression. In this context, the kallikreins have been implicated in the degradation of extracellular matrix (ECM) molecules, the processing of growth factors to their active forms (or inactivation of their regulators) and the activation of other enzymes, including other kallikreins, implicated in prostate cancer (Borgono \& Diamandis 2004, Clements et al. 2004).

Epithelial-mesenchymal transition (EMT) is a crucial event in the progression of cancer to an invasive phenotype. The perturbation of E-cadherin and increased expression of vimentin are two currently accepted characteristics associated with EMT. E-cadherin is downregulated in most epithelial cancers, and can be correlated to higher mobility and invasiveness of tumour cells (Hirohashi \& Kanai 2003). The change in E-cadherin expression observed in many prostate cancer cases can be attributed to methylation of the E-cadherin promoter (Kallakury et al. 2001) or truncation of the protein leading to its inactivation (Davies et al. 2001). In contrast, vimentin expression is usually increased in cells which have undergone EMT and has been characterised in migrating cancer cells (Hendrix et al. 1997, Gilles et al. 1999, Singh et al. 2003). Vimentin is associated with increased in vitro motility of prostate cancer cell lines, and immunohistochemical studies have shown elevated levels of vimentin in the majority of advanced prostate cancer tissues and bone metastases (Lang et al. 2002).

In this study, we sought to determine the relative expression levels of hK4, compared with the more wellknown PSA and $\mathrm{hK} 2$, and to determine its involvement in biological processes associated with cancer progression. We have unequivocally shown, for the first time, a clearly increased expression of $\mathrm{hK} 4$, at the protein level, in cancer compared with benign tissues. This observation not only shows that $\mathrm{hK} 4$ is more cancer specific in expression than PSA or hK2 - thus having potential as a useful diagnostic/prognostic biomarker for prostate cancer - but also suggests that hK4 may play an important functional role in prostate cancer progression. To elucidate such a role, we have characterised PC-3 cells transfected separately with the three prostate-specific kallikreins, $K L K 4, K L K 3 /$ PSA and $K L K 2$. We report that the expression of $\mathrm{hK} 4$ and PSA, but not hK2, promotes cell migration and alters the structure of these cells. E-cadherin expression is lost, while vimentin expression is increased, both hallmarks of EMT-like behaviour. These findings suggest that the prostatic kallikreins, hK4 and PSA, may play a role in the initiation of EMT-like changes in prostate cancer cells and thus are important modulators of prostate cancer progression.

\section{Materials and methods}

\section{Immunohistochemistry}

Formalin-fixed paraffin blocks from prostate tumours $(n=6)$ and benign prostatic hyperplasia $(\mathrm{BPH})(n=6)$ were sectioned $(4 \mu \mathrm{m})$, deparaffinised and rehydrated. After $\mathrm{H}_{2} \mathrm{O}_{2}$ treatment to quench endogenous peroxidase, the sections were incubated overnight with an anti-PSA polyclonal antibody (1:5000 dilution; Dako, Botany, NSW, Australia), anti-hK2 monoclonal antibody (1:700 dilution; clone HK1G 586.1; Hybritech, San Diego, CA, USA), or anti-hK4 polyclonal antibody $\left(1: 250\right.$ dilution) at $4{ }^{\circ} \mathrm{C}$ respectively. The EnVision $^{+}$peroxidase polymer detection system (Dako) was used with 3,3'-diaminobenzidine (DAB; Sigma, Castle Hill, NSW, Australia) as the chromogen. The sections were counterstained with Mayer's haematoxylin. Normal goat serum $(10 \%)$ replaced the primary antibodies as a negative control. The hK4 peptide antibody was generated by immunisation of New Zealand rabbits using a peptide, IINGEDCSPHSQ, designed to the $\mathrm{NH}_{2}$-terminal region of hK4. The production, specificity and characterisation of the hK4 antibody is described elsewhere (Harvey et al. 2003).

\section{Cell lines, expression plasmids and transfection}

PC-3 and LNCaP prostate cancer cell lines (American Type Culture Collection, Rockville, MD, USA), were maintained in RPMI 1640 medium supplemented with $10 \%$ fetal bovine serum (FBS), $2 \mathrm{mM}$ L-glutamine, 100 units $/ \mathrm{ml}$ penicillin $\mathrm{G}$ sodium and $100 \mu \mathrm{g} / \mathrm{ml}$ streptomycin sulphate. For transfection, the entire coding region (pre-pro-enzyme) of $K L K 4, K L K 3$ or $K L K 2$ cDNA was amplified from mRNA extracted 
from $\mathrm{LNCaP}$ cells and inserted into the mammalian expression vector, pcDNA3.1 (Invitrogen, Mt Waverley, Victoria, Australia). PC-3 cells, which do not express $K L K 3$ or $K L K 2$ and express low levels of $K L K 4$ endogenously, were transfected with the individual plasmids (pcDNA3.1:KLK4, pcDNA3.1:KLK3 or pcDNA3.1:KLK2) or an empty vector plasmid (pcDNA3.1:vector only), using the lipid-mediated Lipofectamine 2000 Reagent protocol (Invitrogen). Stably transfected cells were selected in medium containing $100 \mu \mathrm{g} / \mathrm{ml} \mathrm{G418} \mathrm{(Invitrogen)} \mathrm{and} \mathrm{are}$ denoted as PC-3: hK4 (nos. 1-6), PC-3:PSA (nos. 1-7), PC-3: hK2 (nos. 1-7) or PC-3: vector only (nos. 1 and 2) cell lines.

\section{Western blot analysis}

Western blotting was performed with cell lysates collected from several cell clones, while conditioned medium was collected after serum starvation for $48 \mathrm{~h}$ and concentrated 20 -fold. Total protein concentrations were measured by the micro-bicinchoninic assay (BCA; Pierce, Progen, Darra, Queensland, Australia) with BSA standards. An amount of $10 \mu \mathrm{g}$ protein samples was separated on $8-12 \%$ SDS polyacrylamide gels under denaturing conditions, transferred onto nitrocellulose membranes (Schleicher and Schuell; Medos, Mount Waverley, Victoria, Australia) and stained with Ponceau S (Sigma) to ensure that transfer and equal loading had occurred. Membranes were quenched with $5 \%$ skim milk blocking solution before primary antibodies were added and incubated at $4{ }^{\circ} \mathrm{C}$ overnight. Primary antibodies were as follows: hK2-HK1G 586.1 (Hybritech, San Diego, CA, USA), PSA-PSM 773.3.3 (Hybritech) and hK4- $\mathrm{NH}_{2}$-terminal peptide antibody. Horseradish peroxidase-conjugated goat antimouse (hK2 and PSA) or goat antirabbit (hK4) secondary antibodies (Pierce) were applied before the addition of West Femto substrate (Pierce), chemiluminescent exposure to radiographic film and development with a Curix 60 automatic developer (Agfa, Stafford, Queensland, Australia).

\section{RT-PCR and real-time PCR}

Total RNA was extracted with TRI-Reagent (Sigma). cDNA was synthesised using Superscript II (Invitrogen), and RT-PCR and real-time PCR were performed with primers specific to the gene of interest (see figure legends). Real-time PCR was performed on an ABI 7000 Thermal Cycler using the SYBR Green I Dye detection system (Applied Biosystems, Scoresby, Victoria, Australia). Relative levels of gene expression were normalised to $18 \mathrm{~S}$ ribosomal RNA. Each sample was amplified three times in triplicate. Statistical analysis was performed with Student's $t$-test.

\section{Immunofluorescence and confocal microscopy}

Cells were grown on sterile 8- or 16-well glass chamber slides (Medos). Double labelling for actin (tetramethyl rhodamine isothiocyanate (TRITC)-conjugated phalloidin) and vinculin (clone 7F9) was performed with an actin cytoskeleton and focal adhesion staining kit (Chemicon, Boronia, Victoria, Australia). Tubulin was detected with the clone 2G10 antibody (Upstate Biotechnology, Auspep, Parkville, Victoria, Australia). Antibodies against E-cadherin (HECD-1 (extracellular domain) and 4A2C7 (intracellular domain); Zymed, Gymea, NSW, Australia) and vimentin (LN6; Sigma) were used for immunofluorescence. Secondary incubations were performed with AlexaFluor 488 goat antimouse immunoglobulin (Ig)G antibody (Molecular Probes, Bioscientific, Gymea, NSW, Australia). A Leitz fluorescent compound microscope and/or a Leica TCS 4D confocal microscope were used for imaging.

\section{MTT cell proliferation assay}

MTT (3-(4,5-dimethylthiazol-2-yl)-2,5-diphenyltetrazolium bromide) proliferation assays were based on a reported method (Mosmann 1983), with modifications. All cell line clones were used in this assay and assessed every $24 \mathrm{~h}$ for a total of $96 \mathrm{~h}$. Experiments were assayed with 18-24 replicates and repeated three times. Statistical analysis was determined at $96 \mathrm{~h}$ by Student's $t$-test.

\section{In vitro motility and Matrigel invasion assays}

Cell motility was determined by a reported procedure (Saito et al. 1997). PC-3 cell lines $\left(5 \times 10^{4}\right)$ were seeded onto $8 \mu \mathrm{m}$ pore tissue culture inserts (Falcon, BD Biosciences, North Ryde, NSW, Australia) in serumfree medium containing $0.1 \%$ BSA, and allowed to migrate toward the chemoattractant $(20 \% \mathrm{FCS})$ for $24 \mathrm{~h}$ (hK4-expressing cells) or $48 \mathrm{~h}$ (PSA- and hK2expressing cells). Invasion assays were performed as for the motility assays but with growth factor-reduced Matrigel (BD Biosciences) coated on the upper surface of the insert. All assays were performed in duplicate on four occasions, with proliferation over the experimental period taken into account. Statistical analysis was performed by one-way ANOVA with Tukey's post hoc analysis. 


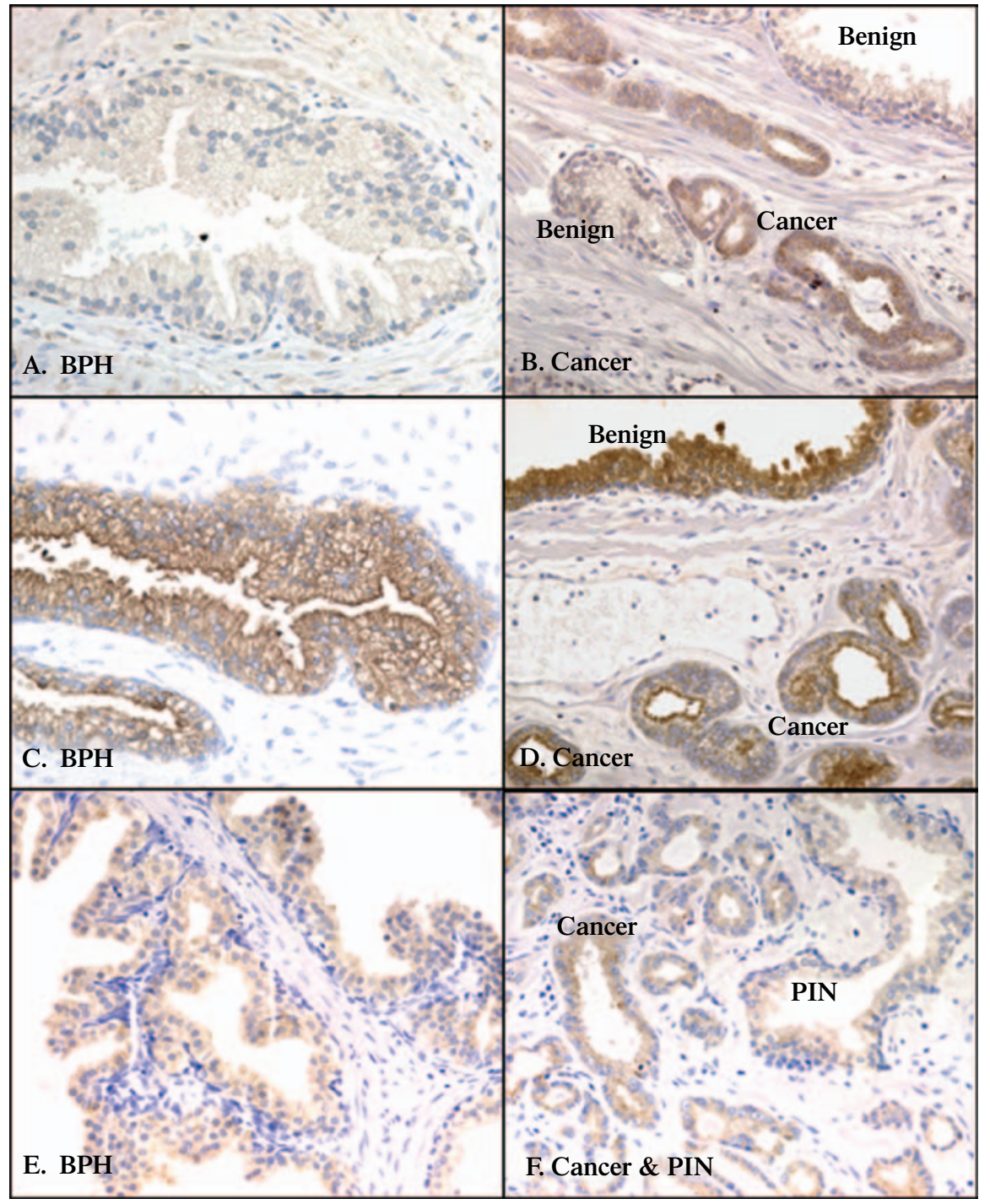

Figure 1 Immunohistochemical staining for hK4, PSA and hK2 in benign prostatic hyperplasia (BPH) and prostate cancer tissue specimens. (A and B) hK4 staining. (C and D) PSA staining. (E and F) hK2 staining. PIN: prostatic intraepithelial neoplasia.

\section{Results}

\section{Immunohistochemistry of kallikrein expression in prostatic tissues}

To validate the previous reports of increased hK4 mRNA in prostate cancer, we first determined the relative expression levels of hK4 (protein) compared with PSA and hK2 in cancer and benign glands. This was done by immunohistochemistry, and the results shown in Fig. 1 (A-F) are representative of the staining patterns observed for all BPH $(n=6)$ and prostate cancer $(n=6)$ tissue sections. As expected, hK4, PSA and $\mathrm{hK} 2$ expression was primarily localised to the cytoplasm of epithelial cells in both benign and cancer glands. Weak staining was observed in the epithelium of the benign glands stained with the hK4 peptide antibody, whereas strong immunoreactivity was found in the cancerous glands (Fig. 1A and B). As previously reported (Darson et al. 1997), the intensity of PSA staining was decreased in prostate cancer in comparison with the BPH tissue sections (Fig. 1C and D). hK2 immunostaining was slightly increased in cancer tissues, again as previously reported (Darson et al. 1999), particularly when compared with the PIN lesion (Fig. 1E and F). No staining was seen in the negative controls (data not shown). 
These data provide the first evidence that $\mathrm{hK} 4$ protein is more abundant in prostate cancer tissue than in benign tissue and raise the question of its cancerrelated functional role.

\section{Establishment of an in vitro kallikrein expression model in stably transfected PC-3 cells}

In order to examine the possible functional effects of hK4 in prostate cancer, we established an in vitro expression model in which PC-3 cells were stably transfected with expression constructs for either $K L K 4$, $K L K 3$ or $K L K 2$. PC-3 cells were chosen for this study because they express little or no PSA, hK2 or hK4. RT-PCR analysis confirmed that the clones transfected with $K L K 4, K L K 3$ or $K L K 2$ were all positive for the expression of their specific genes (Fig. 2A). The native PC-3 cells and vector-only clones were appropriately negative, although low levels of endogenous KLK4 expression were detected in these cells. Western blotting also showed that $\mathrm{hK} 4$, PSA and $\mathrm{hK} 2$ protein was overexpressed in the cell lysate and, as typical of these serine proteases, secreted into the medium when compared with the control native or vector-only cells (Fig. 2B). All clones were analysed for changes in proliferation rates, while a selection of clones (asterisked in Fig. 2A) were chosen for the other functional assays.

\section{Morphology and phenotype of kallikrein- expressing PC-3 cells}

After stable transfection, both the hK4- and PSAexpressing PC-3 cells displayed an elongated/irregular morphology (Fig. 2C). In contrast, cells transfected with the pcDNA3.1:KLK2 or pcDNA3.1: vector-only constructs were similar in structure to the native PC-3 cell line, which had a rounded shape and formed tightly adherent colonies typical of an epithelial, cobblestone appearance.

Subcellular staining of microtubules (green) and actin filaments (red) in each cell line (Fig. 3A) confirmed the small, rounded shape in the majority of native PC-3 cells and those transfected with the empty-vector or pcDNA3.1:KLK2 constructs. The appearance of hK4- and PSA-expressing cell lines was quite distinct from the control cell lines, particularly in the prevalence of small, actin-rich filopodia/ microspikes (Fig. 3A; indicated by arrows). More extensive lamellipodia/membrane ruffling (indicated by asterisks) can be observed in these cells than in the native, vector control or hK2-expressing cell lines. Cells with lamellipodia also had classical tubulin staining - dense within the cell body and weak in the lamella (Fig. 3A).

Furthermore, it was noted that the KLK4- and PSAtransfected PC-3 cells had compromised adhesion to the culture surface, did not display significant cell-tocell contacts and did not grow in distinct colonies. Staining for vinculin (green) and actin (red) (Fig. 3B) confirmed these findings in that the native, vector-only and hK2-expressing PC-3 cell lines revealed distinct focal adhesions with actin and vinculin colocalising to the outer edges of the cell (Fig. 3B; arrowheads). Less concentrated regions of colocalisation were detected in the cells expressing hK4 and PSA.

\section{Cell proliferation is decreased in hK4- expressing PC-3 cells, but not altered in PSA- or hK2-expressing cells}

In three independent assays, the proliferation of the hK4-expressing PC-3 clones was decreased 2-fold at $96 \mathrm{~h}(P<0.01)$ when compared with the parent PC-3 cells (Fig. 4A). No change in the proliferation rate of the PSA- or hK2-expressing clones was observed when compared with control cells at the conclusion of the experimental period.

\section{hK4 and PSA expression in PC-3 cells promotes cell motility, but not cell invasion}

None of the prostatic kallikreins expressed in the PC-3 cells significantly induced in vitro invasion through Matrigel compared with the control cell lines (Fig. 4B). In contrast, hK4-transfected PC-3 cells had increased motility at $24 \mathrm{~h}$ in comparison with the PC-3 native cells $(2.4$-fold increase, $P<0.01)$ and vector-only controls (2.9-fold increase, $P<0.01$ ) (Fig. 4C). PSA expression in PC-3 cells induced a 2.7-fold increase in motility compared with the vector-only control cell lines $(P<0.01)$ and a 1.7 -fold increase in motility compared with the PC-3 native cells at $48 \mathrm{~h}(P<0.01)$ (Fig. 4D). Although no change in motility was seen for the hK2-expressing cells compared with the native cells, there was an increase in migration compared with the vector-only controls, but this was not significant. Interestingly, it is noted that the hK4-expressing cells migrated significantly faster (within $24 \mathrm{~h}$ ) than PC-3 cells expressing PSA and hK2 (measured over $48 \mathrm{~h}$ ).

\section{Expression of E-cadherin is decreased in response to hK4 and PSA expression in PC-3 cells}

These findings suggested that an EMT-like event may have occurred; therefore, the cells were analysed for 
$T$ L Veveris-Lowe, M G Lawrence, $R$ L Collard et al.: Kallikreins and EMT in PC-3 cells
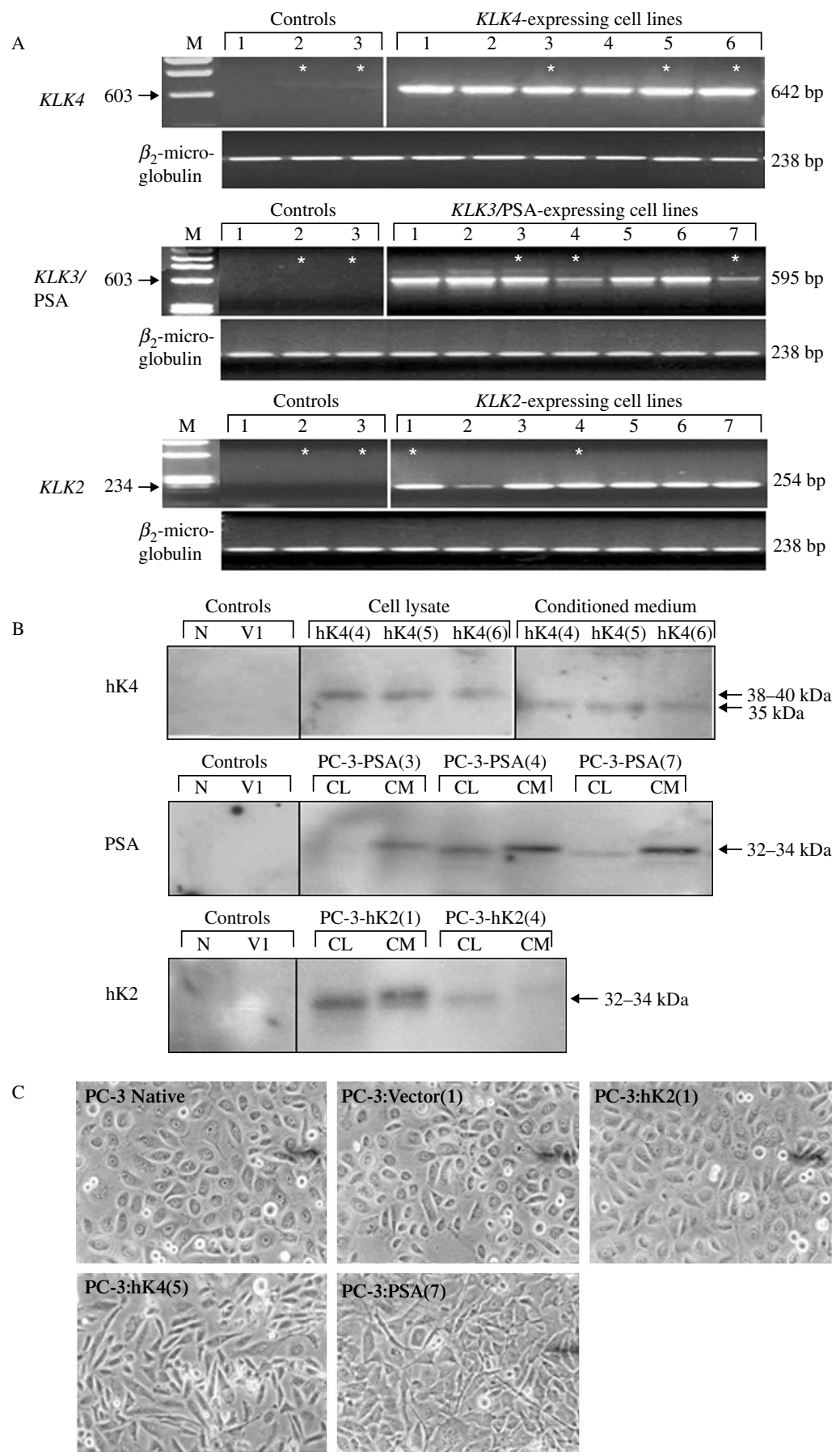

Figure 2 (A) RT-PCR analysis (PCR primers in parentheses) for $K L K 4$ (forward (for): $5^{\prime}$-atggccacagcaggaaatccc- $3^{\prime}$, reverse (rev): 5'-caaggccctgcaagtacccg-3'), KLK3/PSA (for: 5'-atcgaattcgcacccggagagctgtgt-3', rev: 5'-ctgagggtgaacttgcgcacac-3'), KLK2 (for: 5'-tgaagcatcaaagccttagac-3', rev: 5'-ctcagactaagctctagcacac-3'), and $\beta 2$-microglobulin housekeeping gene (for: 5'tgaattgctatgtgtctgggt-3', rev: 5'-cctccatgatgctgcttacat-3'). All clones expressing KLK4 (nos. 1-6), KLK3/PSA (nos. 1-7), KLK2 (nos. 1-7), an untransfected/native PC-3 control (lane 1) and two different vector-only cell lines (nos. 1 and 2 ) (lanes 2 and 3 ) were analysed. Transfected clones used for functional analysis are indicated by asterisk. (B) Western blots for hK4, PSA and hK2 on representative PC-3 clones, using cell lysates $(C L)$ and conditioned medium (CM). Each panel includes cell lystates from two control cell lines (N: PC-3 native; V1: PC-3:vector-only (no. 1)). (C) Phase-contrast micrographs of the PC-3 native cells and representatives of the PC-3: vector-only (no. 1), PC-3:hK4 (no. 5), PC-3:PSA (no. 7) and PC-3:hK2 (no. 1) clones. 
A
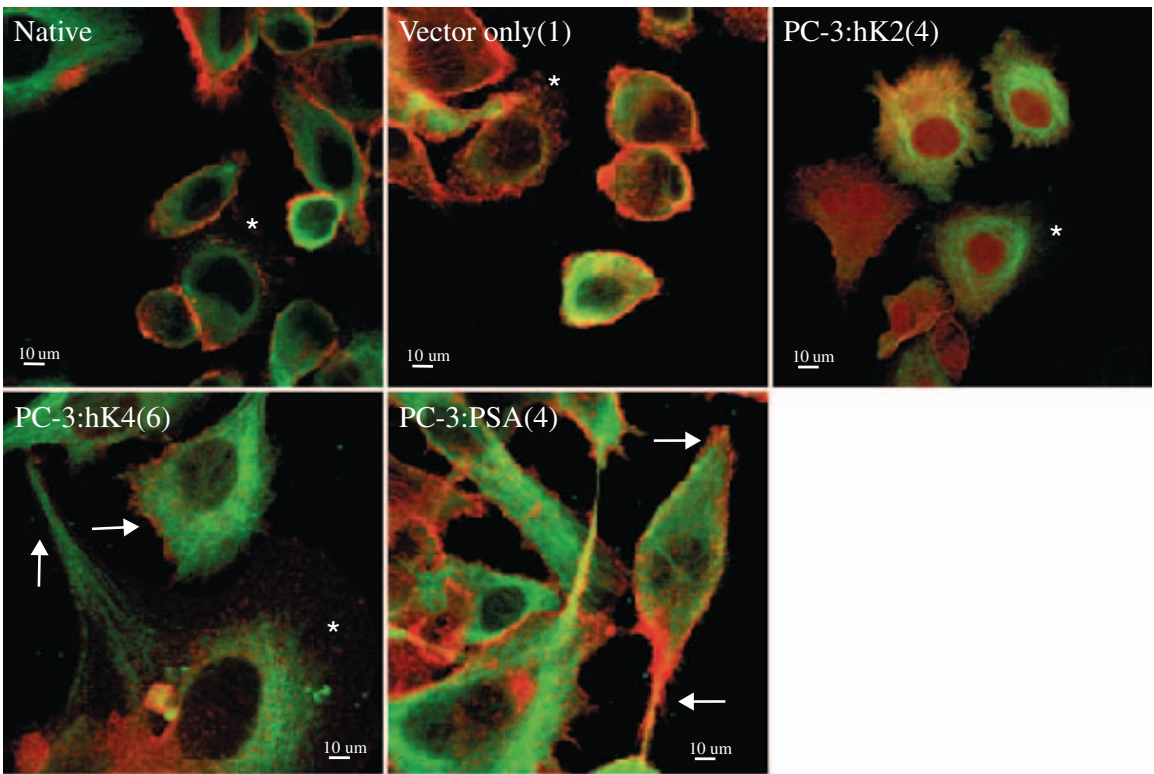

B
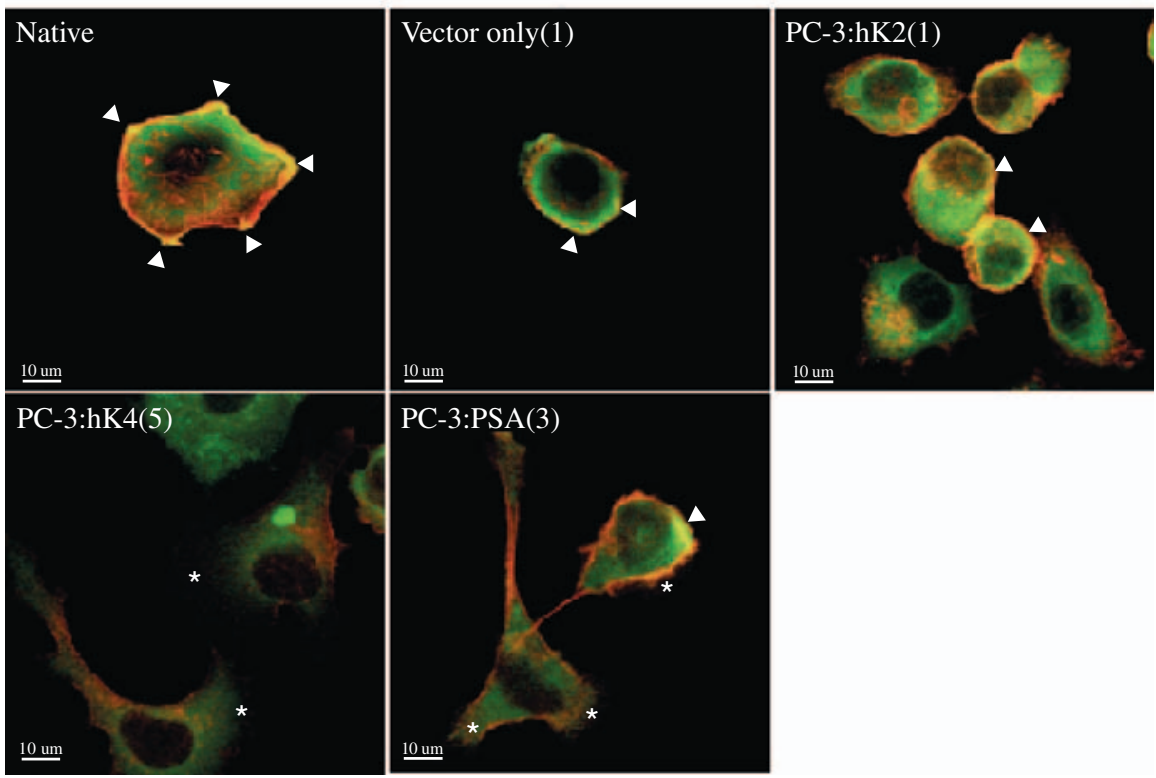

Figure 3 (A) Representative clones from the native PC-3 cells, PC-3:vector-only (no. 1), PC-3:hK2 (no. 4), PC-3:hK4 (no. 6) and PC-3:PSA (no. 4), illustrating merged confocal images of tubulin (green) and actin (red) staining. Lamellipodia/membrane ruffles are indicated by asterisk, while arrows denote filopodia/microspikes. (B) Representative confocal images of cells stained for vinculin (green) and actin (red). Prominent areas of colocalisation are indicated by arrowheads. Major lamellipodia at the leading edge of PC-3:hK4 (no. 5) and PC-3:PSA (no. 3) cells are indicated by asterisk.

changes in E-cadherin and vimentin, key markers of epithelial and mesenchymal phenotypes respectively (Savanger 2001). Little or no E-cadherin staining was observed in the hK4- and PSA-expressing cells with an antibody (HECD-1) specific to the E-cadherin ectodomain, while typical patterns of membrane staining at cell-cell contacts were observed in the control cell lines and cells expressing hK2 (Fig. 5A). Identical patterns of expression were found with a second antibody directed to the intracellular domain of E-cadherin (4A2C7; data not shown), indicating that the entire E-cadherin protein was lost from the hK4- and PSA-expressing cell lines. This suggests that the downregulation of E-cadherin may be transcriptional. Real-time PCR analysis for E-cadherin gene expression showed a highly significant 7 -fold decrease in 
A

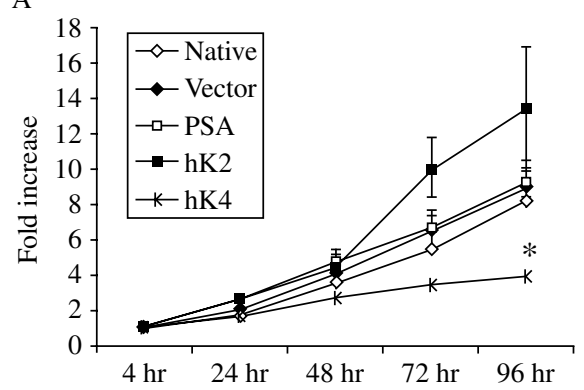

$\mathrm{C}$

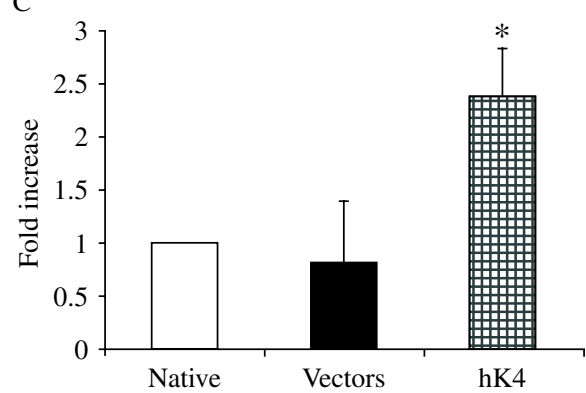

B

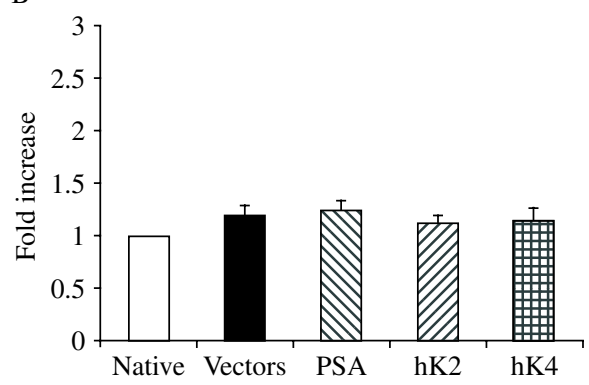

$\mathrm{D}$

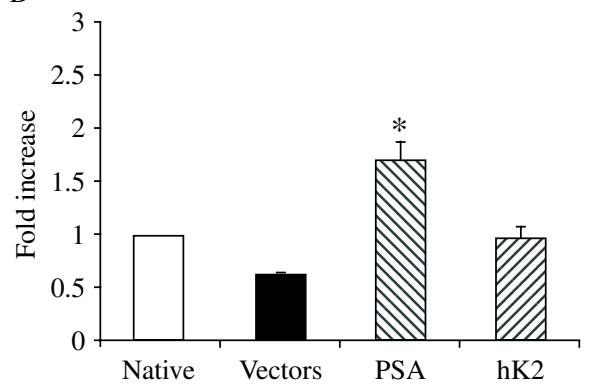

Figure 4 (A) Proliferation assay: graph of the mean data generated from three separate proliferation experiments and averaged for each clone type. Statistical significance was analysed by Student's $t$-test. (B) Matrigel invasion assay: fold changes for PSA-, hK2- and hK4-expressing PC-3 cells compared with the PC-3 native cells (set at 1 ) and vector-only controls $(n=4,48$ h). (C) Motility assay: fold changes for hK4-expressing PC-3 cells compared with the PC-3 native (set at 1) and vector-only controls $(n=3,24 \mathrm{~h})$. (D) Motility assay: fold changes for PSA- and hK2-expressing cells compared with the PC-3 native (set at 1$)$ and vector-only controls $(n=4,48 \mathrm{~h})$. All motility and invasion assays were performed with $5 \times 10^{4}$ cells per well, in duplicate.

Statistical analysis was performed by one-way ANOVA and Tukey's post hoc analysis; S.E.M. are indicated by bars. *Statistically significant difference from controls $(P<0.01)$.

PSA-expressing clones and a 10-fold decrease for hK4expressing clones (Fig. 5B) compared with the PC-3 native cell line $(P<0.01)$ and the vector-only controls $(P<0.05)$. No change was seen in the hK2-expressing cells compared with the control cell lines $(P=0.14)$. As expected for an EMT event, increased staining of vimentin was evident in the cells exhibiting a decrease in E-cadherin (Fig. 5A). These results were confirmed by real-time PCR, demonstrating that vimentin gene expression was elevated in PSA-expressing clones (3.7-fold; $P<0.01$ ) and PC-3 cells expressing hK4 (2.3-fold) (Fig. 5B), although this increase for hK4 did not reach statistical significance $(P=0.27)$. Again, there was no significant change in the non-EMT-like, hK2-expressing cells.

\section{Discussion}

The immunohistochemical data presented here have established, for the first time, that $\mathrm{hK} 4$ protein is more highly expressed in cancer tissues than benign tissues. We have shown that hK4 is localised to the cytoplasm of glandular epithelial cells, using an
$\mathrm{NH}_{2}$-terminal peptide antibody, which detects fulllength hK4. This is consistent with previous reports suggesting that the mRNA levels for KLK4 are increased in prostate cancer tissues (Obiezu et al. 2002, Xi et al. 2004) and strongly implicating hK4 as a possible diagnostic/prognostic marker in the progression of prostate cancer. One study has reported no change in hK4 protein, immunohistochemically, in prostate cancer tissues compared with benign tissues (Obiezu et al. 2002), but it is unclear how many samples were analysed in this report, and this finding could not be confirmed in our study. Both PSA and $\mathrm{hK} 2$ are being used clinically as diagnostic markers for prostate cancer (Borgono \& Diamandis 2004, Clements et al. 2004) despite their recognised shortcomings. The addition of hK4 as an adjunct biomarker deserves further testing and consideration.

Strikingly, the data presented here also implicate hK4 and PSA, but not hK2, in an epithelialmesenchymal transition (EMT) process in prostate cancer cells. EMT is a critical event in the progression of most cancers, as epithelial cells lose their characteristic phenotype and dedifferentiate (Thiery 2002). 
A

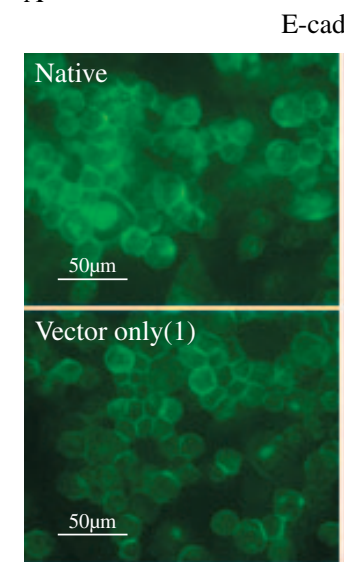

E-cadherin

Vimentin
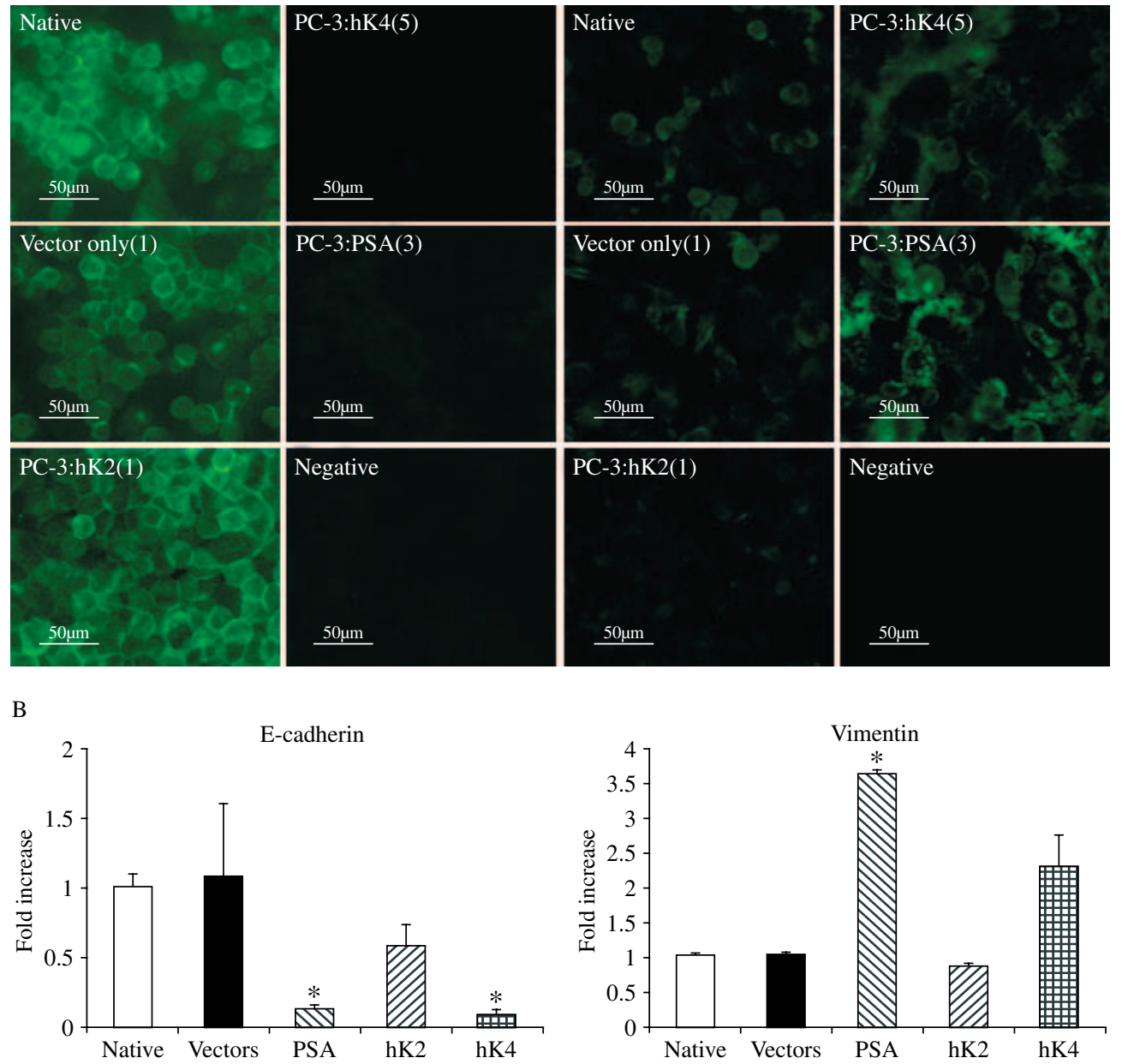

Figure 5 (A) Representative immunofluorescence images of PC-3 cells stained for E-cadherin (with HECD-1 extracellular domain antibody) and vimentin in the control cell lines, PC-3 native and PC-3 vector-only (no. 1), and PC-3:hK2 (no. 1), PC-3:hK4 (no. 5) and PC-3:PSA (no. 3) cells. The negative control for untransfected PC-3 cells is shown. (B) Real-time PCR for E-cadherin (forward (for): 5'-gcccatttcctaaaaacctgg-3', reverse (rev): 5'-ttggatgacacagcgtgagag-3') and vimentin (for: 5'aacaccctgcaatctttc-3', rev: 5'-ccatttcctccttcatattc-3'), using the PCR primers indicated in parentheses on PC-3 vector-only (nos. 1 and 2), PC-3:PSA (nos. 3, 4 and 7), PC-3:hK2 (nos. 1 and 4) and PC-3:hK4 (nos. 3, 5 and 7) cell lines, performed in triplicate. Gene expression was normalised to $18 \mathrm{~S}$ ribosomal RNA (for: 5'-ttcggaactgaggccatgat-3', rev: 5'-cgaacctccgactttcgttc-3' primers). All assays were analysed by Student's $t$-test. S.E.M. are indicated by bars. ${ }^{*}$ Statistically significant difference from native PC-3 cells. ( $P$ values $\mathrm{E}$-cadherin: native vs hK4/PSA $=P<0.01$; vectors vs $\mathrm{hK} 4 / \mathrm{PSA}=P<0.05$; hK2, $P=0.14$. $P$ values vimentin: native vs $\mathrm{PSA}=P<0.01$; native vs hK4, $P=0.27$.)

Specifically, migrating epithelial cells often appear spindle-shaped, with loss of cell polarity, reduced cell-cell adhesion and rearrangement of the cytoskeleton (Savanger 2001, Thiery 2002). Observations about the functional effects of these kallikreins are based on the altered morphology and increased motility displayed by the hK4- and PSA-expressing, but not hK2-expressing, PC-3 cells with a concomitant decrease in E-cadherin and increase in vimentin, two well-known EMT markers of epithelial and mesenchymal origin respectively (Savanger 2001).

Although increased levels of serum PSA are associated with metastatic cancer, it is now well documented that PSA gene and protein expression in prostatic cells decreases as tumours become more aggressive and increasingly undifferentiated (Darson et al. 1997, 1999, Tremblay et al. 1997). The reasons for this loss of PSA expression within malignant cells are 
unclear. Of interest, while the proven metastasispromoting gene, hepsin, has been associated with overexpression in prostate cancer tissues (Klezovitch et al. 2004, Stephan et al. 2004), its expression has been reported to be lower or even lost in metastatic cancer, suggesting that these genes could exert their effects in the early stages of invasive carcinoma rather than at the metastatic lesion (Vasioukhin 2004). We propose that this may be the case for PSA in prostate cancer progression with a subsequent loss of effects such as the suggested putative antiangiogenic and apoptotic roles (Balbay et al. 1999, Fortier et al. 1999, 2003), which would prevail in the normal functioning of the prostate. Contrary to the immunologic pattern presented by PSA in malignancy, we and several others have observed increased $K L K 2 / \mathrm{hK} 2$ and $K L K 4 / \mathrm{hK} 4$ expression in prostatic tumours (Herrala et al. 2001, Xi et al. 2004). This might suggest that $\mathrm{hK} 4$ and $\mathrm{hK} 2$ are more highly associated with malignancy than PSA. Furthermore, our migration studies have indicated that while PSA increased motility in PC-3 cells over $48 \mathrm{~h}$, the cells expressing hK4 migrated significantly faster, suggesting that hK4 may be more important than PSA in the motility of cancer cells and ultimately cancer progression.

PC-3 cells were chosen for this study because they express little or no PSA, hK2 or hK4, unlike LNCaP cells. Although PC-3 cells also do not express $\alpha$-catenin (Morton et al. 1993), a protein associated with linkage of E-cadherin to the actin cytoskeleton, other molecules, such as vinculin, have been shown to take the place of $\alpha$-catenin, thereby allowing re-establishment of functional, cadherin-mediated cell adhesion (Hazan et al. 1997). Functional consequences of E-cadherin perturbations have also been reported in DU145 prostate cancer cells (Chunthapong et al. 2004), despite the variable expression of $\alpha$-catenin in this cell line. Nonetheless, since E-cadherin was completely lost from the hK4- and PSA-expressing PC-3 cells, post-translational modifications and/or the lack of $\alpha$-catenin are unlikely to be the mechanisms involved in the transcriptional decrease of E-cadherin mRNA and subsequent loss of protein.

As serine proteases, the kallikreins serve posttranslationally to cleave specific polypeptide precursors to their bioactive forms, thereby indirectly initiating a range of cellular responses (Borgono \& Diamandis 2004, Clements et al. 2004). This study has indicated that such changes may include growth regulation and EMT. A role for prostatic kallikreins in growth regulation has been predicated on the basis of in vitro biochemical analyses of PSA, hK2 and hK4. For instance, all three kallikreins have been shown to degrade insulin-like growth (IGF) factor-binding proteins (IGFBPs) (Clements et al. 2004, Matsumura et al. 2004). This may then increase the bioavailability of the IGFs and therefore their mitogenic action in the prostate microenvironment. From these findings, PSA has been suggested to contribute to cancer cell growth, but, consistent with the findings of Denmeade et al. (2003), this was not demonstrated in our study. Furthermore, hK4 decreased PC-3 cell proliferation in this study, suggesting that it may not have a proliferative role, but instead may activate inhibitors of mitogenic factors or increase apoptosis.

Although it is unclear what level of active protease is secreted by these cells, since chromogenic substrate analysis was inconclusive (data not shown), a number of factors involved in the EMT process are known kallikrein substrates. Increasing levels of transforming growth factor (TGF) $\beta$ in patients with metastatic prostate cancer have been correlated with the expression of PSA in serum (Adler et al. 1999). The latent (pro-) form of TGF $\beta$ is known to be activated by PSA (Killian et al. 1993) and, by prediction, also by hK4 (Matsumura et al. 2004). TGF $\beta$ has a number of recognised effects on prostate cancer cells, including activation of cytoskeletal modulators, such as the GTPase, RhoA (Bhowmick et al. 2001), and thus rearrangement of the actin cytoskeleton and induction of cell migration. TGF $\beta$ can also downregulate E-cadherin expression through the transcription factors, Snail and Slug, and promote hypermethylation of the E-cadherin gene, leading to loss of the epithelial phenotype (Savanger 2001, Thiery 2002).

PSA can also activate epidermal growth factor (EGF) (Clements et al. 2004), which can increase cell motility by altering cell polarisation and morphology in fibroblasts (Ware et al. 1998). EGF (Unlu \& Leake 2003a), as well as TGF $\beta$ (Unlu \& Leake 2003b), can upregulate urokinase plasminogen activator (uPA) expression and the invasive ability of prostate cancer cells. Recombinant $\mathrm{hK} 4$ and $\mathrm{hK} 2$ have also been shown to activate the precursor of single-chain uPA (Takayama et al. 2001). Thus, although PC-3 cells intrinsically produce high levels of uPA, this enzyme is unlikely to be the primary mechanism for PSA- or hK4induced EMT. First, the EMT-like phenotype is not induced by hK2, a known activator of uPA; second, PSA does not activate uPA (Frenette et al. 1997), yet it induces EMT; third, neither PSA nor hK4 induced the PC-3 cells to invade through artificial ECM in vitro above the control cell lines; and, finally, no EMT-like effect was observed in the vector-only controls.

In summary, we have developed in vitro expression models for the prostatic kallikreins, PSA, hK2 and 
hK4. Characterisation of the phenotype and genotype of these cells clearly indicates, for the first time, that $\mathrm{hK} 4$, as well as PSA, has properties that facilitate the progression of prostate cancer cells by modulating regulators of the cytoskeleton and cellular adhesion and migration processes, all hallmarks of the EMT-like processes indicative of aggressive disease.

\section{Acknowledgements}

We thank Dr Tracey Harvey for preparing the pcDNA3.1:KLK4 construct and Dr Christina Theodoropolous for assistance with confocal microscopy. We also wish to thank Stephen Mikolajczyk (Beckman Coulter Inc, San Diego, CA, USA) for the gift of the research-use anti-hk2 monoclonal antibody.

\section{Funding}

This work was supported by the National Health and Medical Research Council of Australia (grant no. 101202). $\mathrm{T}$ Veveris-Lowe and $\mathrm{R}$ Collard received Wesley Research Institute and Queensland Cancer Fund postgraduate scholarships respectively. The authors declare that there is no conflict of interest that would prejudice the impartiality of this scientific work.

\section{References}

Adler HL, McCurdy MA, Kattan MW, Timme TL, Scardino PT \& Thompson TC 1999 Elevated levels of circulating interleukin- 6 and transforming growth factor-betal in patients with metastatic prostatic carcinoma. Journal of Urology 161 182-187.

American Cancer Society 2004 Cancer statistics presentation download www.cancer.org/docroot/pro/content/ pro_1_1_Cancer_Statistics_2004_presentation.asp.

Balbay MD, Juang P, Llansa N, Williams S, McConkey D, Fidler IJ \& Pettaway CA 1999 Stable transfection of human prostate cancer cell line PC-3 with prostatespecific antigen induces apoptosis both in vivo and in vitro. Proceedings of the American Association for Cancer Research 40 225-226.

Bhowmick NA, Ghiassi M, Bakin A, Aakre M, Lundquist CA, Engel ME, Arteaga CL \& Moses HL 2001

Transforming growth factor-betal mediates epithelial to mesenchymal transdifferentiation through a RhoAdependent mechanism. Molecular Biology of the Cell 12 27-36.

Borgono CA \& Diamandis EP 2004 The emerging roles of human tissue kallikreins in cancer. Nature Reviews. Cancer 4 876-890.

Chunthapong J, Seftor EA, Khalkhali-Ellis Z, Seftor REB, Amir S, Lubaroff DM, Heidger PM \& Hendrix MJC 2004
Dual roles of E-cadherin in prostate cancer invasion. Journal of Cellular Biochemistry 91 649-661.

Clements JA, Willemsen NM, Myers SA \& Dong Y 2004 The tissue kallikrein family of serine proteases: functional roles in human disease and potential as clinical biomarkers. Critical Reviews in Clinical Laboratory Sciences 41 265-312.

Darson MF, Pacelli A, Roche P, Rittenhouse HG, Wolfert RL, Young CY, Klee GG, Tindall DJ \& Bostwick DG 1997 Human glandular kallikrein 2 (hK2) expression in prostatic intraepithelial neoplasia and adenocarcinoma: a novel prostate cancer marker. Urology 49 857-862.

Darson MF, Pacelli A, Roche P, Rittenhouse HG, Wolfert RL, Saeid MS, Young CY, Klee GG, Tindall DJ \& Bostwick DG 1999 Human glandular kallikrein 2 expression in prostate adenocarcinoma and lymph node metastases. Urology 53 939-944.

Davies G, Jiang WG \& Mason MD 2001 Matrilysin mediates extracellular cleavage of E-cadherin from prostate cancer cells: a key mechanism in hepatocyte growth factor/scatter factor-induced cell-cell dissociation and in vitro invasion. Clinical Cancer Research 7 3289-3297.

Denmeade SR, Litvinov I, Sokoll LJ, Lilja H \& Isaacs JT 2003 Prostate-specific antigen (PSA) protein does not affect growth of prostate cancer cells in vitro or prostate cancer xenografts in vivo. The Prostate 56 45-53.

Diamandis EP, Yousef GM, Clements J, Ashworth LK, Yoshida S, Egelrud T, Nelson PS, Shiosaka S, Little S, Lilja H, Stenman UH, Rittenhouse HG \& Wain H 2000 New nomenclature for the human tissue kallikrein gene family. Clinical Chemistry $\mathbf{4 6}$ 1855-1858.

Fortier AH, Nelson BJ, Grella DK \& Holaday JW 1999 Antiangiogenic activity of prostate-specific antigen. Journal of the National Cancer Institute 91 1635-1640.

Fortier AH, Holaday JW, Liang H, Dey C, Grella DK, Holland-Linn J, Vu H, Plum SM \& Nelson BJ 2003 Recombinant prostate specific antigen inhibits angiogenesis in vitro and in vivo. The Prostate $\mathbf{5 6}$ 212-219.

Frenette G, Tremblay RR, Lazure C \& Dube JY 1997 Prostatic kallikrein hK2, but not prostate-specific antigen (hK3), activates single-chain urokinase-type plasminogen activator. International Journal of Cancer 71 897-899.

Gilles C, Polette M, Zahm JM, Tournier JM, Volders L, Foidart JM \& Birembaut P 1999 Vimentin contributes to human mammary epithelial cell migration. Journal of Cell Science 112 (Pt 24) 4615-4625.

Haese A, Graefen M, Steuber T, Becker C, Pettersson K, Piironen T, Noldus J, Huland H, Lilja H \& Huland E 2001 Human glandular kallikrein 2 levels in serum for discrimination of pathologically organ-confined from locally-advanced prostate cancer in total PSA-levels below $10 \mathrm{ng} / \mathrm{ml}$. The Prostate 49 101-109.

Haese A, Graefen M, Becker C, Noldus J, Katz J, Cagiannos I, Kattan M, Scardino PT, Huland E, Huland H \& Lilja H 
2003 The role of human glandular kallikrein 2 for prediction of pathologically organ confined prostate cancer. The Prostate 54 181-186.

Harvey TJ, Dong Y, Bui L, Jarrott R, Walsh T \& Clements JA 2003 Production and characterization of antipeptide kallikrein 4 antibodies. Use of computer modeling to design peptides specific to kallikrein 4. Methods in Molecular Medicine 81 241-254.

Hazan RB, Kang L, Roe S, Borgen PI \& Rimm DL 1997 Vinculin is associated with the E-cadherin adhesion complex. Journal of Biological Chemistry 272 32448-32453.

Hendrix MJ, Seftor EA, Seftor RE \& Trevor KT 1997 Experimental co-expression of vimentin and keratin intermediate filaments in human breast cancer cells results in phenotypic interconversion and increased invasive behavior. American Journal of Pathology 150 483-495.

Herrala AM, Porvari KS, Kyllonen AP \& Vihko PT 2001 Comparison of human prostate specific glandular kallikrein 2 and prostate specific antigen gene expression in prostate with gene amplification and overexpression of prostate specific glandular kallikrein 2 in tumor tissue. Cancer 92 2975-2984.

Hirohashi S \& Kanai Y 2003 Cell adhesion system and human cancer morphogenesis. Cancer Science 94 575-581.

Jemal A, Tiwari RC, Murray T, Ghafoor A, Samuels A, Ward E, Feuer EJ \& Thun MJ 2004 Cancer statistics, 2004. CA: A Cancer Journal for Clinicians 54 8-29.

Kallakury BV, Sheehan CE, Winn-Deen E, Oliver J, Fisher HA, Kaufman RP Jr \& Ross JS 2001 Decreased expression of catenins (alpha and beta), p120 CTN, and E-cadherin cell adhesion proteins and E-cadherin gene promoter methylation in prostatic adenocarcinomas. Cancer 92 2786-2795.

Killian CS, Corral DA, Kawinski E \& Constantine RI 1993 Mitogenic response of osteoblast cells to prostate-specific antigen suggests an activation of latent TGF-beta and a proteolytic modulation of cell adhesion receptors.

Biochemical and Biophysical Research Communications 192 940-947.

Klezovitch O, Chevillet J, Mirosevich J, Roberts RL, Matusik RJ \& Vasioukhin V 2004 Hepsin promotes prostate cancer progression and metastasis. Cancer Cell 6 185-195.

Lang SH, Hyde C, Reid IN, Hitchcock IS, Hart CA, Bryden AA, Villette JM, Stower MJ \& Maitland NJ 2002 Enhanced expression of vimentin in motile prostate cell lines and in poorly differentiated and metastatic prostate carcinoma. The Prostate 52 253-263.

Matsumura M, Bhatt AS, Andress D, Clegg N, Takayama TK, Craik CS \& Nelson PS 2005 Substrates of the prostate-specific serine protease prostase/KLK4 defined by positional-scanning peptide libraries. The Prostate $\mathbf{6 2}$ $1-13$.

Morton RA, Ewing CM, Nagafuchi A, Tsukita S \& Isaacs WB 1993 Reduction of E-cadherin levels and deletion of the alpha-catenin gene in human prostate cancer cells. Cancer Research 53 3585-3590.

Mosmann T 1983 Rapid colorimetric assay for cellular growth and survival: application to proliferation and cytotoxicity assays. Journal of Immunological Methods $\mathbf{6 5}$ 55-63.

Obiezu CV, Soosaipillai A, Jung K, Stephan C, Scorilas A, Howarth DH \& Diamandis EP 2002 Detection of human kallikrein 4 in healthy and cancerous prostatic tissues by immunofluorometry and immunohistochemistry. Clinical Chemistry 48 1232-1240.

Partin AW, Hanks GE, Klein EA, Moul JW, Nelson WG \& Scher HI 2002a Prostate-specific antigen as a marker of disease activity in prostate cancer. I. Oncology (Huntington) 16 1024-1038.

Partin AW, Hanks GE, Klein EA, Moul JW, Nelson WG \& Scher HI $2002 b$ Prostate-specific antigen as a marker of disease activity in prostate cancer. II. Oncology (Huntington) 16 1218-1224.

Saito K, Oku T, Ata N, Miyashiro H, Hattori M \& Saiki I 1997 A modified and convenient method for assessing tumor cell invasion and migration and its application to screening for inhibitors. Biological and Pharmaceutical Bulletin 20 345-348.

Savanger P 2001 Leaving the neighborhood: molecular mechanisms involved during epithelial-mesenchymal transition. BioEssays 23 912-923.

Singh S, Sadacharan S, Su S, Belldegrun A, Persad S \& Singh G 2003 Overexpression of vimentin: role in the invasive phenotype in an androgen-independent model of prostate cancer. Cancer Research 63 2306-2311.

Stephan C, Yousef GM, Scorilas A, Jung K, Jung M, Kristiansen G, Hauptmann S, Kishi T, Nakamura T, Loening SA \& Diamandis EP 2004 Hepsin is highly over expressed in and a new candidate for a prognostic indicator in prostate cancer. Journal of Urology 171 187-191.

Takayama TK, McMullen BA, Nelson PS, Matsumura M \& Fujikawa K 2001 Characterization of hK4 (prostase), a prostate-specific serine protease: activation of the precursor of prostate specific antigen (pro-PSA) and single-chain urokinase-type plasminogen activator and degradation of prostatic acid phosphatase. Biochemistry 40 15341-15348.

Thiery JP 2002 Epithelial-mesenchymal transitions in tumour progression. Nature Reviews. Cancer 2 442-454.

Tremblay RR, Deperthes D, Tetu B \& Dube JY 1997 Immunohistochemical study suggesting a complementary role of kallikreins hK2 and hK 3 (prostate-specific antigen) in the functional analysis of human prostate tumors. American Journal of Pathology 150 455-459.

Unlu A \& Leake RE $2003 a$ The effect of EGFR-related tyrosine kinase activity inhibition on the growth and invasion mechanisms of prostate carcinoma cell lines. International Journal of Biological Markers $\mathbf{1 8}$ $139-146$. 
Unlu A \& Leake RE 2003b Transforming growth factor betal stimulates urokinase plasminogen activator system on prostate cancer cells. International Journal of Biological Markers 18 147-151.

Vasioukhin V 2004 Hepsin paradox reveals unexpected complexity of metastatic process. Cell Cycle 3 1394-1397.

Ware MF, Wells A \& Lauffenburger DA 1998 Epidermal growth factor alters fibroblast migration speed and directional persistence reciprocally and in a matrixdependent manner. Journal of Cell Science 111 2423-2432.

Xi Z, Klokk TI, Korkmaz K, Kurys P, Elbi C, Risberg B, Danielsen H, Loda M \& Saatcioglu F 2004 Kallikrein 4 is a predominantly nuclear protein and is overexpressed in prostate cancer. Cancer Research 64 2365-2370. 
